\title{
On the Choice of Shear Correction Factor in Sandwich Structures
}

\author{
VICTOR BIRMAN* \\ Engineering Education Center, University of Missouri-Rolla, \\ 8001 Natural Bridge Road, St. Louis, MO 63121, USA
}

CHARles W. BERT

Aerospace and Mechanical Engineering, The University of Oklahoma, Norman, OK 73019-1052, USA

\begin{abstract}
The first-order shear deformation theory (FSDT) is a relatively simple tool that has been found to yield accurate results in the non-local problems of sandwich structures, such as buckling and free vibration. However, a key factor in practical application of the theory is determination of the transverse shear correction factor $(K)$, which appears as a coefficient in the expression for the transverse shear stress resultant. The physical basis for this factor is that it is supposed to compensate for the FSDT assumption that the shear strain is uniform through the depth of the cross section. In the present paper, the philosophies and results of $K$ determination for homogeneous rectangular cross sections are first reviewed, followed by a review and discussion for the case of sandwich structures. The analysis presented in the paper results in the conclusion that $K$ should be taken equal to unity, as a first approximation, for both two-skin as well as for multi-skin sandwich structures.
\end{abstract}

\section{INTRODUCTION}

$\mathbf{T}$ HE PURPOSE OF using sandwich construction in the first place is to provide a stronger and stiffer structure for the same weight, or conversely a lighter structure to carry the same load, as a homogeneous or compact-laminate flexural member. The main load-carrying portions are the stiff facings and the purpose of the core is to serve as a spacer or separator to keep the facings apart at a large distance from the neutral surface. The facings and core play analogous roles to the flanges and web of an

*Author to whom correspondence should be addressed.

Journal of SANDWich StRUCtURES AND MATERIALs, Vol. 4-January 2002 
I-beam. Consequently, the maximum normal stresses (tension and compression) are in the facings but the core carries the brunt of the transverse shear stresses. The transverse shear stresses are negligible in the facings as are normal stresses in the core. The transverse shear strains resulting from the transverse shear stresses produce additional deflection and flexibility, which usually must be taken into account. Thus, there is a need for knowledge of the factor $K$.

\section{TRANSVERSE SHEAR CORRECTION FACTOR FOR HOMOGENEOUS SECTIONS}

The first theory to include both transverse shear deformation and rotatory inertia apparently was due to Bresse in 1859 [1]. However, the concept of $K$ was introduced by Timoshenko in 1921 [2]. Originally, he proposed the value of $2 / 3$, based on the maximum transverse shear strain, which occurs at the neutral surface. One year later, based on Filon's experimental results, Timoshenko proposed a value of 0.889 [3]. Based on elementary transverse shear theory, Goens [4] derived a value of $5 / 6$, approximately 0.833 , which is the value widely used today. Also, although he did not use the terminology of $K$, Reissner's well-known shear deformable plate theory [5] is tacitly consistent with the $5 / 6$ value.

Apparently, Olsson [6] in 1935 was the first to incorporate the effect of Poisson's ratio ( $v$ ) on $K$. His result, as well as later work of Cowper [7] and Kaneko [8] can be expressed as

$$
K=(5 / 6)(1+v) /(1+\mathrm{a} v)
$$

where $a=5 / 8,11 / 12$, and 5/6, according to [6-8], respectively. Independently, Hutchinson [9] and Wittrick [10] obtained $K=5 /(6-v)$. Kaneko compared the various predictions with experimental data for materials with $v$ between 0.13 and 0.38 and found that a value of $a=5 / 6$ gave the best agreement.

The analyses mentioned above were based on static considerations only. Apparently, Mindlin [11] in 1951 was the first to predict $K$ based on a dynamic analysis. One prediction utilized matching pure thickness-shear waves and gave $K=\pi^{2} / 12$, or approximately 0.822 . His other prediction was based on short-wavelength flexural waves and gave an implicit result that depended on Poisson's ratio.

In a very important analysis, Hutchinson and Zillmer [12] used an exact series solution of the dynamic elasticity equations and worked backward to obtain the value of $K$ to match the frequencies with those of FSDT. They showed that $K$ depends not only upon $v$, but also on mode number, 
width/depth and depth/length ratios. In a recent paper [13], Hutchinson derived a new formula for $K$ using a comparison between the first-order Timoshenko beam solution for long wavelengths and the variational Hellinger-Reissner formulation for a "best" guess for the stress and displacement fields. One of the interesting conclusions from this paper was that for a rectangular cross section, $K$ is a function of the aspect ratio.

The present paper illustrates several methods of evaluation of the shear correction factor applicable to sandwich structures. It is shown that a family of methods yields the value of this factor equal to unity, irrespectively of the stiffness and geometry of the structure. Note that this value of $K$ has been adopted in numerous studies utilizing FSDT. Another method, originally proposed by Bert et al. [14], results in the factor that depends only on geometry (thickness of the facings and the core) of a symmetrically laminated sandwich structure. However, the other family of methods outlined below yields the factor that is affected by both geometry as well as the stiffnesses of the facings and core. Surprisingly, as is shown in the examples, the value of this shear correction factor approaches zero, as the ratio of the shear stiffness of the facings to that of the core is increased.

\section{SHEAR CORRECTION FACTOR FOR SANDWICH STRUCTURES}

\section{Methods Yielding the Shear Correction Factor Equal to Unity}

\section{METHOD BASED ON THE COMPARISON OF NATURAL FREQUENCIES}

$\mathrm{Yu}$ published an accurate analysis for the shear correction factor of a three-layered sandwich plate based on the comparison of the fundamental frequencies obtained by the theory of elasticity solution and by the FSDT model of the structure [15]. It was shown that for a typical sandwich plate the value of this factor approaches unity.

\section{METHOD BASED ON THE COMPARISON OF AVERAGE STRESSES THROUGHOUT THE CROSS SECTION}

This is a new method that has been developed by the authors. This method is based on equating the average shear stress in the beam cross section based on mechanics of materials to the average stress evaluated for the Timoshenko beam (FSDT). The factor $K$ derived as shown in Appendix A is

$$
K=-D / \int_{-h / 2}^{h / 2}\left[\int_{-h / 2}^{z} Q_{11} z d z\right] d z
$$


where $h$ is the thickness of the beam, and $Q_{11}$ and $D$ are a transformed reduced stiffness of the corresponding layer and the bending stiffness, respectively. For a single isotropic layer, Equation (2) yields $K=1$, i.e. a value of the shear correction factor that is higher than the typically suggested values of $5 / 6$ or $\pi^{2} / 12$.

Consider now a symmetric sandwich beam where $h_{c}, h_{f}$, and $h$ denote the thicknesses of the core, the facings, and the beam, respectively. Evaluating the numerator and denominator of the expression in the right side of Equation (2) yields the identical result:

$$
D=-\int_{-h / 2}^{h / 2}\left[\int_{-h / 2}^{z} Q_{11} z d z\right] d z=(1 / 12)\left[Q_{11}^{f}\left(h^{3}-h_{c}^{3}\right)+Q_{11}^{c} h_{c}^{3}\right]
$$

where the indices $f$ and $c$ refer to the facings and core, respectively. Obviously, $K=1$.

\section{METHOD BASED ON THE MINIMIZATION OF THE}

\section{QUADRATIC ERROR OF THE SHEAR STRESS}

This approach is based on the requirement that the square of the integral difference between the shear stresses obtained by mechanics of materials and FSDT must be minimum. The shear correction factor serves as an independent variable. Accordingly,

$$
(\partial / \partial K)\left[\int_{-h / 2}^{h / 2}\left[G_{i} Q_{x} /\left(K A_{55}\right)+\left(\int_{-h / 2}^{z} Q_{11} z d z\right) Q_{x} / D\right] d z\right]^{2}=0
$$

where $A_{55}=\Sigma G_{i} h_{i}$ is the stiffness obtained as a sum of the transverse shear moduli multiplied by the thickness of the corresponding layers and $Q_{x}$ is the transverse shear stress resultant.

It can be shown that Equation (4) yields Equation (2), i.e. $K$ for a symmetric sandwich beam, according to this method, is equal to unity.

\section{Method Based on Modeling the Sandwich Structure as a Discrete Mass System}

This method is based on representing the sandwich panel as a discretemass system where the facings replaced with lumped masses are connected through a shear spring that models the core. The natural frequency of this system is compared to the expression for the fundamental frequency in pure shear motion obtained for the Timoshenko beam. The comparison yields the following expression for the shear correction factor of a sandwich with 
two identical facings [14]:

$$
K=2 J / B \rho_{f a} h_{c}^{2}
$$

where $J$ is the composite mass moment of inertia per unit length of the beam, $B$ is the beam width, and $\rho_{f a}$ is the mass of one facing, including the adhesive layer, per unit surface area.

If the sandwich structure is symmetric, Equation (5) can be simplified as follows. The mass moment of inertia per unit length is equal to

$$
J=\left(B \rho_{f a} / 2\right)\left(h_{c}+h_{f}\right)^{2}
$$

The substitution of Equation (6) into Equation (5) and simplifications result in

$$
K=\left(1+h_{f} / h_{c}\right)^{2}
$$

Note that the factor $K$ obtained by Equation (7) does not depend on the stiffness of the facings or the core. It varies from unity for very thin facings to larger values for the case where the thicknesses of each of the facings and core are of the same order.

\section{Methods that Yield the Shear Correction Factor as a Function of the Stiffness and Geometry}

\section{METHOD BASED ON THE COMPARISON OF THE}

\section{SHEAR STRAIN ENERGIES}

A closed-form solution for the shear correction factor of a laminated beam developed by Bert and Gordaninejad [16] based on the comparison of the shear strain energy for the actual beam to the counterpart for an equivalent Timoshenko beam yields

$$
\left.K=\left(A D-B^{2}\right)^{2} /\left[A_{55} \int_{-h / 2}^{h / 2}(A b-B a)^{2} / G\right) d z\right]
$$

where $A, B$, and $D$ are extensional, coupling, and bending stiffnesses, respectively, $G=G(z)$ is the transverse shear modulus, and

$$
\{a, b\}=\int_{-h / 2}^{z}\{1, z\} Q_{11}(z) d z
$$


Note that a similar result is also available from independently derived expressions for the shear correction factor published by Chou [17] (symmetric only) and Whitney [18]. The approach to derivation of these expressions was also based on a comparison of the shear strain energies.

Obviously, Equation (8) is also applicable to sandwich structures. For example, for a symmetric sandwich beam having a compact cross section, transformations yield

$$
K=D^{2} /\left[A_{55}\left(2 b_{1}+b_{2}\right)\right]
$$

where

$$
\begin{gathered}
D=(1 / 12)\left[E_{f}\left(h^{3}-h_{c}^{3}\right)+E_{c} h_{c}^{3}\right] \\
A_{55}=2 G_{f} h_{f}+G_{c} h_{c} \\
b_{1}=\left(E_{f}^{2} / 4 G_{f}\right)\left[(8 / 15)(h / 2)^{5}-(h / 2)^{4}\left(h_{c} / 2\right)+(2 / 3)(h / 2)^{2}\left(h_{c} / 2\right)^{3}-\left(h_{c} / 2\right)^{5} / 5\right] \\
b_{2}=\left\{E_{c}^{2}\left(h_{c} / 2\right)^{5} / 5+E_{c}\left(h_{c} / 2\right)^{3}\left[E_{f}\left(h_{c}^{2}-h^{2}\right)-E_{c} h_{c}^{2}\right] / 6\right. \\
\left.+\left[E_{f}\left(h_{c}^{2}-h^{2}\right)-E_{c} h_{c}^{2}\right]^{2} h_{c} / 32\right\} /\left(2 G_{c}\right)
\end{gathered}
$$

The shear correction factor given by Equation (8) or Equation (10) depends both on the thickness and on the stiffness of the facings and the core. In particular, if $h_{f}=h_{c}$, this factor is [16]:

$$
K=(5 / 6)(26 \alpha+1)^{2} g /\left\{(1+2 g)\left[102 \alpha^{2}+g\left(120 \alpha^{2}+20 \alpha+1\right)\right]\right\}
$$

where $\alpha=E_{f} / E_{c}$ and $g=G_{f} / G_{c}$. However, if the core has both axial and shear stiffnesses that are very small, compared to those of the facings, both $\alpha$ and $g$ are much larger than unity. In this case, Equation (12) degenerates to $K=2.35 / \mathrm{g}$. Obviously, if $g$ is large, the value of $K$ becomes small, approaching zero as a limit. Note that if $\alpha$ is very large but $g$ remains finite, the factor $K$ is close to the values that could be anticipated for sandwich structures. For example, if $g=1, K=0.850$, as follows from Equation (12). However, as $g$ increases to 10, the value of $K$ drops to 0.206.

It is interesting to note that Equation (8) could also be obtained from the requirement that the squared error in the shear strain energy due to a replacement of the energy based on the theory of elasticity with the energy of the Timoshenko beam should be minimal. Therefore, we can conclude that the energy based static approach predicts very low values of $K$ if the shear stiffness of the core is a small fraction of that of the facings. 


\section{METHOD BASED ON THE COMPARISON}

\section{OF THE AVERAGE STRAINS}

A different approach is to compare the integrals of the strain throughout the depth of the beam cross section generated using mechanics of materials and the shear deformation theory which is equivalent to a comparison of the average strain. The derivation shown in Appendix B yields

$$
K=-D h /\left\{A_{55} \int_{-h / 2}^{h / 2}\left[\int_{-h / 2}^{z} Q_{11} z d z\right] / G_{i} d z\right\}
$$

It is easy to show that in the case of a single layer, $K=1$.

For a sandwich beam, Equation (13) becomes

$$
\begin{aligned}
K= & (2 / 3)\left\{Q_{11}^{f}\left[1-\left(h_{c}^{*}\right)^{3}\right]+Q_{11}^{c}\left(h_{c}^{*}\right)^{3}\right\} \\
& /\left\{[ G _ { f } ( 1 - h _ { c } ^ { * } ) + G _ { c } h _ { c } ^ { * } ] \left[Q_{11}^{f}\left[2 / 3-h_{c}^{*}+\left(h_{c}^{*}\right)^{3} / 3\right] / G_{f}+Q_{11}^{f} h_{c}^{*}\left[1-\left(h_{c}^{*}\right)^{2}\right] / G_{c}\right.\right. \\
& \left.\left.+(2 / 3) Q_{11}^{c}\left(h_{c}^{*}\right)^{3} / G_{c}\right]\right\}
\end{aligned}
$$

where $h_{c}^{*}=h_{c} / h$.

It is obvious that if the shear stiffness of the core approaches zero, the shear correction factor becomes close to zero as well. Note that if the facings are very thin, $K$ approaches unity. If the in-plane axial stiffness of the core is negligible, i.e. $Q_{11}^{c}=0$, Equation (14) becomes

$$
\begin{aligned}
& K=(2 / 3)\left[1-\left(h_{c}^{*}\right)^{3}\right] /\{\left(1-h_{c}^{*}+h_{c}^{*} / g\right)\left[2 / 3-h_{c}^{*}+\left(h_{c}^{*}\right)^{3} / 3\right. \\
&\left.+g h_{c}^{*}\left[1-\left(h_{c}^{*}\right)^{2}\right]\right\}
\end{aligned}
$$

Note that if $g$ is very large, i.e. the core has a low stiffness compared to the facings, the value of $K$ approaches zero.

\section{Numerical Examples and Discussion}

Six methods for the evaluation of the shear correction factor have been discussed in the paper. Three of these methods yield the value of this factor equal to unity, irrespectively of geometry and stiffnesses of the sandwich components. Therefore, in these examples, we compare the factors obtained by the other three methods: modeling the sandwich structure as a discrete mass system (Equation 7), method based on the comparison of the shear 
strain energies (Equations 10,12), and the method based on the comparison of the average strains (Equation 14). Two different sandwich types are considered. The first sandwich, referred to as Type 1, was analyzed in [19]: $E_{f}=2.83 \mathrm{GPa}, G_{f}=5.74 \mathrm{MPa}, E_{c}=5.61 \mathrm{MPa}, G_{c}=4.49 \mathrm{MPa}$. The facing and core thicknesses analyzed in [19] were $h_{f}=1.59 \mathrm{~mm}$ and $h_{c}=28.58 \mathrm{~mm}$. The second case considered for Type 1 sandwich is with $h_{f}=h_{c}=10 \mathrm{~mm}$, while the total sandwich thickness is not altered. Type 2 sandwich is a typical shipbuilding configuration with E-glass/vinyl ester facings and a balsa core. The properties of the facings and the core for Type 2 are: $E_{f}=24.4 \mathrm{GPa}, G_{f}=2.89 \mathrm{GPa}[20]$ and $E_{c}=2.41 \mathrm{GPa}, G_{c}=0.103 \mathrm{GPa}$ [21]. Two geometries considered for this beam were $h_{f}=5 \mathrm{~mm}, h_{c}=20 \mathrm{~mm}$ and $h_{f}=h_{c}=10 \mathrm{~mm}$.

The values of $K$ obtained by three methods are listed in Table 1. As follows from this Table, changes of geometry result in very large variations of the shear correction factor obtained by modeling the sandwich structure as a discrete mass system and by the method based on the comparison of the shear strain energies. The factor obtained by the method based on the comparison of the average strains appears little affected by geometry. However, this factor reduces to zero if the stiffness of the core is low.

This discussion illustrates that three methods yielding the shear correction factor as a function of geometry and stiffness of the sandwich structure are unacceptable at certain geometries and at a low value of the core shear stiffness. Considering the requirement that a method for the shear correction factor should be universal, the above-mentioned methods can be used for the analysis of sandwich structures with caution, keeping in mind their limitations.

Table 1. Values of the shear correction factor obtained for two different sandwich types as functions of geometry and method of evaluation of $K$.

\begin{tabular}{lccc}
\hline $\begin{array}{l}\text { Sandwich type } \\
\text { and geometry }\end{array}$ & $\begin{array}{c}\text { Discrete mass } \\
\text { system }\end{array}$ & $\begin{array}{c}\text { Shear strain } \\
\text { energy }\end{array}$ & $\begin{array}{c}\text { Average } \\
\text { shear strain }\end{array}$ \\
\hline $\begin{array}{c}\text { Type } 1 \\
h_{f}=1.59 \mathrm{~mm} \\
h_{c}=28.58 \mathrm{~mm}\end{array}$ & 1.114 & 0.951 & 0.986 \\
$\begin{array}{c}\text { Type } 1 \\
h_{f}=h_{c}=10 \mathrm{~mm}\end{array}$ & 4.0 & & \\
$\begin{array}{c}\text { Type } 2 \\
h_{f}=5 \mathrm{~mm} \\
h_{c}=20 \mathrm{~mm}\end{array}$ & 1.563 & 0.00473 & 0.960 \\
$\begin{array}{c}\text { Type } 2 \\
h_{f}=h_{c}=10 \mathrm{~mm}\end{array}$ & & 0.105 & 0.124 \\
\hline
\end{tabular}


Three other methods yielding the shear correction factor equal to unity could be criticized on the basis of the fact that if the sandwich structure degenerates to a single isotropic layer, the factor should be equal to $5 / 6,2 / 3$ or $\pi^{2} / 12$. However, it should be noted that a difference between the results obtained for moderately thick structures using the values of $K$ in the range between $2 / 3$ and 1.0 is relatively small. Therefore, it is suggested to employ the factor $K=1$ in the analysis of sandwich structures, particularly, if the core stiffness is low.

\section{Proof that $K=1$ for a Symmetrically Laminated Multi-Skin Sandwich Structure}

As indicated above, the value $K=1$ is appropriate for the analysis of sandwich structures with two facings. In particular, this value was derived based on a comparison of the average shear stresses that yields Equation (2). As shown in Appendix A, Equation (2) is derived without a reference to the number of skins in a sandwich structure. It is also noted that the method based on the minimization of the quadratic error of the shear stress yields the same equation.

It is important to extrapolate the analysis to multi-skin sandwich structures that have recently attracted increasing interest due to their high strength, stiffness, and damage tolerance. It is shown in this section that the shear correction factor given by Equation (2) is equal to unity in case of a multi-skin symmetrically laminated sandwich beam.

Consider a couple of identical layers that are symmetric relative to the sandwich middle axis, as shown in Figure 1. The functions $b$ for each of these layers, as defined by Equation (9), are

$$
\begin{aligned}
& b_{a}=Q_{11}^{a}\left(z^{2}-z_{1}^{2}\right) / 2 \\
& b_{b}=Q_{11}^{a}\left(z_{2}^{2}-z_{1}^{2}\right) / 2+Q_{11}^{b}\left(z^{2}-z_{1}^{2}\right) / 2=Q_{11}^{a}\left(z^{2}-z_{1}^{2}\right) / 2
\end{aligned}
$$

where $Q_{11}^{a}=Q_{11}^{b}$.

Note that for each layer $c$ located between layers $a$ and $b$ the contribution proportional to $Q_{11}^{a}$ is

$$
b_{c}=Q_{11}^{a}\left(z_{2}^{2}-z_{1}^{2}\right) / 2
$$

The contribution of a layer $d$ located at $z>z_{1}$ and proportional to $Q_{11}^{a}$ is

$$
b_{d}=Q_{11}^{a}\left(z_{2}^{2}-z_{1}^{2}\right) / 2+Q_{11}^{b}\left(z_{1}^{2}-z_{2}^{2}\right) / 2=0
$$




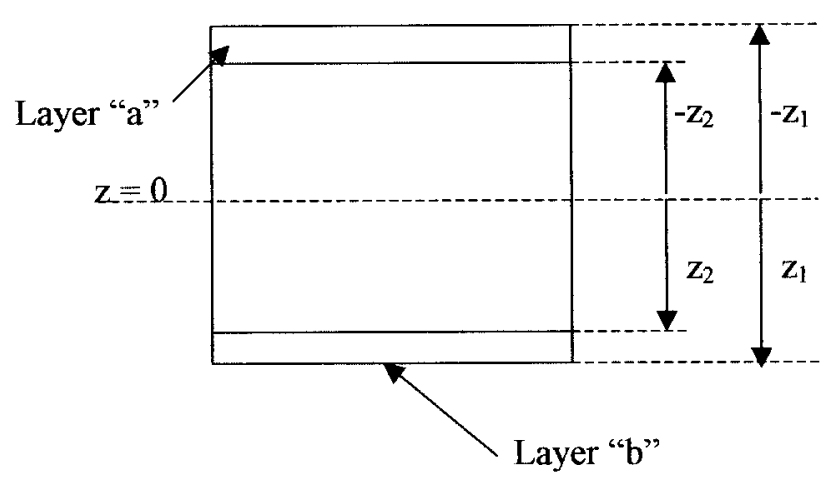

Figure 1. Symmetric couple of identical layers $\left(Q_{11}^{a}=Q_{11}^{b}\right)$.

Now it is possible to calculate the contribution of all layers to the denominator in the right side of Equation (2) that is proportional to $Q_{11}^{\mathrm{a}}$ :

$$
C(a, b)=\int_{-h / 2}^{h / 2}\left(b_{a}+b_{b}+b_{c}+b_{d}\right) d z=-(2 / 3) Q_{11}^{a}\left(z_{1}^{3}-z_{2}^{3}\right)
$$

Note that this contribution is associated only with layers $a$ and $b$, as reflected in notation $C(a, b)$. It is immediately evident that the contribution of layers $a$ and $b$ to the bending stiffness $D$ in the numerator of Equation (2) is equal to $-C(a, b)$. Therefore, summing the contributions of all symmetric couples of layers in all skins one obtains $K=1$.

\section{CONCLUSIONS}

Six methods of the evaluation of the shear correction factor for sandwich structures have been compared. Three of these methods based on modeling the sandwich structure as a discrete mass system, the comparison of the shear strain energies, and the comparison of the average shear strains lack universality, i.e. they can be applied only to selected ranges of geometry and material properties. Three other methods based on the comparison of natural frequencies, the comparison of the average shear stresses and the minimization of the quadratic error of the shear stresses yield $K=1$, irrespectively of geometry and material properties. Moreover, as was proven in the paper, based on the comparison of the average shear stress or the minimization of the quadratic error in this stress, $K=1$ for a multi-skin symmetrically laminated sandwich beam. Therefore, it is recommended to 
use $K=1$, as a first approximation, for the analysis of two-skin and multiskin sandwich structures, unless other universal methods yielding a different value or expression for $K$ are suggested.

\section{ACKNOWLEDGEMENT}

The research of the first author was supported by the Office of Naval Research (Contract N00014-00-1-0323). The program manager is Dr. Yapa Rajapakse.

\section{APPENDIX A}

\section{Derivation of $K$ Based on the Comparison of Average Stresses Throughout the Cross Section}

The theory of elasticity yields the following expression for the transverse shear stress

$$
\tau_{x z}=-(\partial / \partial x) \int \sigma_{x} d z
$$

where $x$ and $z$ are the axial and through-the-thickness coordinates, respectively, $\sigma_{x}$ is the axial stress, and the limits of integration vary from the surface of the beam $z=-h / 2$ to an arbitrary value of $z$.

The constitutive relation for the axial stress in a symmetrically laminated beam subjected to bending is

$$
\sigma_{x}=Q_{11} z \kappa
$$

where $\kappa$ is the beam axis curvature.

The substitution of Equation (A2) into Equation (A1) and using the relationship between the transverse shear stress resultant and the curvature

$$
Q_{x}=\partial M_{x} / \partial x=D \partial \kappa \partial x
$$

yields the shear stress

$$
\tau_{x z}=-\left[\int Q_{11} z d z\right] Q_{x} / D
$$

The stress given by Equation (A4) is derived based on mechanics of materials, without a reference to the shear strain distribution throughout 
the depth of the beam. Now the shear stress will be obtained based on FSDT. The transverse shear stress resultant is given by

$$
Q_{x}=K A_{55} \varepsilon_{x z}
$$

Therefore, the shear stress can be represented by

$$
\tau_{x z}=G_{i} \varepsilon_{x z}=G_{i} Q_{x} /\left(K A_{55}\right)
$$

The shear correction factor is obtained from the requirement that the average shear stresses based on Equations (A4) and (A6) must be equal. This yields

$$
K=-D / \int_{-h / 2}^{h / 2}\left[\int_{-h / 2}^{z} Q_{11} z d z\right] d z
$$

\section{APPENDIX B}

\section{Derivation of $K$ Based on the Comparison of the Average Strains}

For a symmetric unit-width sandwich beam, the integrals of the strain throughout the depth of the cross section are

$$
A_{1}=-\int_{-h / 2}^{h / 2}\left[\int_{-h / 2}^{z} Q_{11} z d z\right] Q_{x} /\left(G_{i} D\right) d z \quad \text { (mechanics of materials) }
$$

and

$$
A_{2}=Q_{x} h /\left(K A_{55}\right) \quad \text { (shear deformation theory) }
$$

respectively.

Equating $A_{1}$ and $A_{2}$, one obtains

$$
K=-D h /\left\{A_{55} \int_{-h / 2}^{h / 2}\left[\int_{-h / 2}^{z} Q_{11} z d z\right] / G_{i} d z\right\}
$$

\section{REFERENCES}

1. Bresse, J.A.C. (1859). Cours de Mecanique Analytiquee. Paris: Mallet-Bachelier.

2. Timoshenko, S.P. (1921). On the correction for shear of the differential equation for transverse vibrations of prismatic bars. Philosophical Magazine, Ser. 6, 41: 742-746.

3. Timoshenko, S.P. (1922). On the transverse vibrations of bars of uniform cross-section. Philosophical Magazine, Ser. 6, 43: 125-131. 
4. Goens, E. (1931). Uber die Bestimmung des Elastizitatsmodule von Staben mit Hilfe von Biegungsschwingungen. Annalen der Physik, Ser. 5, 11: 649-678.

5. Reissner, E. (1945). The effect of transverse shear deformation on the bending of elastic plates. ASME Journal of Applied Mechanics, 12: A69-A77.

6. Olsson, R.G. (1935). Zur Berechnung der Frequenz der Transversalschwingungen des prismatischen Stabes. Zeitschrift fur angewandtle Mathematik und Mechanik, 15: 245.

7. Cowper, G.R. (1966). The shear coefficient in Timoshenko's beam theory. ASME Journal of Applied Mechanics, 33: 335-340.

8. Kaneko, T. (1975). On Timoshenko's correction for shear in vibrating beams. Journal of Physics, Ser. D, 8: 1927-1936.

9. Hutchinson, J.R. (1984). Vibrations of thick free circular plates, exact versus approximate solutions. ASME Journal of Applied Mechanics, 51: 581-585.

10. Wittrick, W.H. (1987). Analytical, three-dimensional elasticity solutions to some plate problems, and some observations on mindlin's plate theory. International Journal of Solids and Structures, 23: 441-464.

11. Mindlin, R.D. (1951). Influence of rotatory inertia and shear on flexural motions of isotropic, elastic plates. ASME Journal of Applied Mechanics, 18: 31-38.

12. Hutchinson, J.R. and Zillmer, S.D. (1986). On the transverse vibration of beams of rectangular cross-sections. ASME Journal of Applied Mechanics, 53: 39-44.

13. Hutchinson, J.R. (2001). Shear coefficients for Timoshenko beam theory. ASME Journal of Applied Mechanics, 68: 87-92.

14. Bert, C.W., Wilkins, D.J., Jr. and Crisman, W.C. (1967). Damping in sandwich beams with shear-flexible cores. ASME Journal of Engineering for Industry, 89B: 662-670.

15. Yu, Y.-Y. (1959). Simple thickness-shear modes of vibration of infinite sandwich plates. ASME Journal of Applied Mechanics, 26: 679-681.

16. Bert, C.W. and Gordaninejad, F. (1983). Transverse shear effects in bimodular composite laminates. Journal of Composite Materials, 17: 282-298.

17. Chou, T.S. (1971). On the propagation of flexural waves in an orthotropic laminated plate and its response to an impulsive load. Journal of Composite Materials, 5: 306-319.

18. Whitney, J.M. (1973). Shear correction factors for orthotropic laminates under shear load. ASME Journal of Applied Mechanics, 40: 302-304.

19. Rebello, C.A., Bert, C.W. and Gordaninejad, F. (1983). Vibration of bimodular sandwich beams with thick facings: a new theory and experimental results. Journal of Sound and Vibration, 90: 381-397.

20. Gibson, R.F. (1994). Principles of Composite Materials Mechanics. New York: McGrawHill.

21. Allen, H.L. (1969). Analysis and Design of Structural Sandwich Panels. Oxford: Pergamon Press. 